\title{
Constraints on diffuse neutrino emission from the Galactic Plane with 7 years of IceCube data
}

\author{
The IceCube Collaboration ${ }^{\dagger}$, \\ ${ }^{\dagger}$ http://icecube.wisc.edu/collaboration/authors/icrc17_icecube \\ E-mail: analysis@icecube.wisc.edu
}

\begin{abstract}
The origins of high-energy astrophysical neutrinos measured by the IceCube Neutrino Observatory remain a mystery despite extensive searches for multimessenger correlations. In particular, no point sources have been identified so far. However, a likely source for diffuse neutrino emission are cosmic-ray interactions in the Galactic plane. Due to the excellent pointing of their track-like signature, muon-neutrino-induced muons are an excellent channel for measuring spatial correlations. Two methods were developed to test for a spatially extended flux from the entire Galactic plane. Both methods are maximum likelihood fits, one binned and the other unbinned, and using different background estimation methods. We consider two templates for Galactic neutrino emission based primarily on gamma-ray observations and models that cover a wide range of possibilities. We present constraints from seven years of IceCube Neutrino Observatory muon data on the neutrino flux coming from the Galactic plane.
\end{abstract}

Corresponding authors: Christian Haack ${ }^{* 1}$, Jon Dumm ${ }^{2}$

${ }^{1}$ III. Physikalisches Institut B, RWTH Aachen University

${ }^{2}$ Oskar Klein Centre and Dept. of Physics, Stockholm University

35th International Cosmic Ray Conference - ICRC2017-

10-20 July, 2017

Bexco, Busan, Korea

\footnotetext{
${ }^{*}$ Speaker.
} 


\section{Introduction}

The high-energy gamma-ray sky is dominated by diffuse emission from our Galaxy, being the first discovered steady source of astrophysical gamma rays [1]. Cosmic-ray interactions with ambient interstellar gas are the dominant production mechanism for high-energy gamma rays in the plane of the Galaxy via the decay of neutral pions. Diffuse neutrinos from the plane of the Galaxy are expected from these same interactions via decay of charged pions. We perform searches for diffuse neutrino emission based on models constructed from gamma-ray observations.

The IceCube collaboration has reported the detection of a flux of high-energy $(>10 \mathrm{TeV})$ astrophysical neutrinos $[2,3,4]$. In a combined fit to multiple data sets, the flux was characterized from $25 \mathrm{TeV}$ to $2.8 \mathrm{PeV}$ as a power law with spectral index $2.50 \pm 0.09$ [5]. A recent analysis of only muon neutrinos in the northern sky, with a higher energy threshold of $191 \mathrm{TeV}$ and sensitive up to $8.3 \mathrm{PeV}$, yields a harder spectral index of $2.13 \pm 0.13$ [4]. This difference could indicate either anisotropy or a spectral break. Both explanations are consistent with the existence of a relatively soft Galactic contribution dominating in the southern sky in addition to a harder, isotropic extragalactic component. The astrophysical neutrino signal is so far compatible with isotropy despite searches for anisotropy including point sources (e.g. [6]).

In the three-year sample of IceCube high-energy starting events (HESE), some correlation with the Galactic plane was observed with a chance probability of 2.8\% [3]. In [7], the authors explore the addition of an energy cut in the public HESE data ${ }^{1}$ and report $\mathrm{a}>3 \sigma$ a posteriori correlation with the Galactic plane in the HESE arrival distribution for an energy cut $>100 \mathrm{TeV}$.

Additionally, the ANTARES neutrino detector has performed a search for muon neutrinos in the region of the Galactic ridge. For the case of a neutrino flux that extends into the GeV energy range as an $E^{-2.5}$ power law, they set a per-flavor flux normalization (at $100 \mathrm{GeV}$ ) upper limit of $1.9 \times 10^{-17} \mathrm{GeV}^{-1} \mathrm{~cm}^{-2} \mathrm{~s}^{-1} \mathrm{sr}^{-1}$ in the region Galactic longitude $|l|<40^{\circ}$ and Galactic latitude $|b|<3^{\circ}$ (encompassing $0.145 \mathrm{sr}$ ). [8].

Using IceCube muon samples, we search for diffuse Galactic neutrino emission using two parallel maximum likelihood methods sensitive primarily in the outer Galactic plane region (the northern sky). The first method, denoted as the unbinned method, is an extension of a commonly used point-source analysis. It employs an unbinned maximum likelihood method to search for an anisotropic pattern in the neutrino arrival directions. The second method, denoted as the binned method, is an extension of the diffuse astrophysical neutrino measurement [4]. It is based on binned multi-dimensional templates of all contributing flux components from atmospheric and astrophysical neutrinos. This method inherently includes systematic uncertainties but requires higher purity with respect to atmospheric muon background and is thus restricted to the northern hemisphere.

\section{Models of Galactic Neutrino Emission}

Models for diffuse gamma-ray production are driven by current gamma-ray observations. Thanks to the high-precision measurements of the Fermi-LAT satellite (see [9] for a summary), accurate models of the gamma-ray diffuse emission are available. From the pionic component of these models neutrino predictions can also be made. We discuss two spatial models used in our

\footnotetext{
${ }^{1}$ https://icecube.wisc.edu/science/data
} 

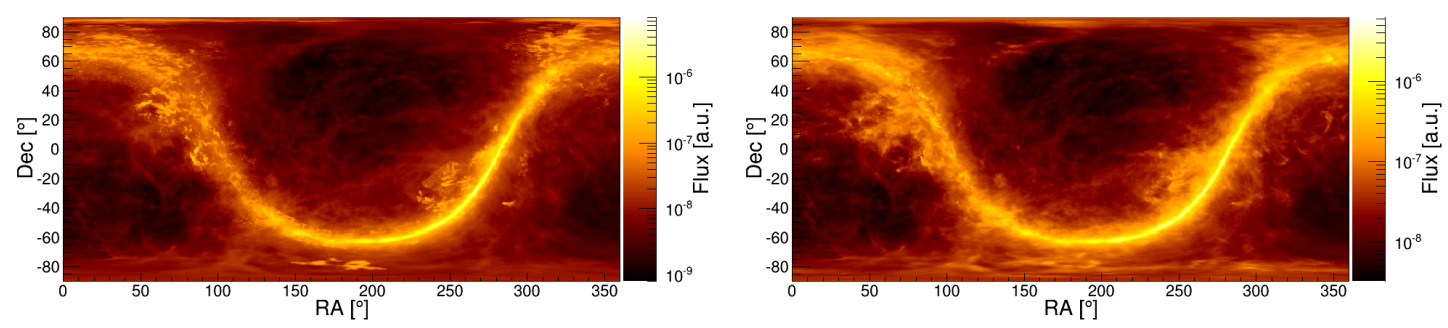

Figure 1: Two models for diffuse Galactic neutrino production described in section 2 are shown: (a) KRA- $\gamma$ (50 PeV cutoff), and (b) Fermi-LAT $\pi^{0}$-decay map.

analysis that span a robust range of possibilities for Galactic neutrino emission: the Fermi-LAT pion-decay template [9] and the KRA- $\gamma$ (50 PeV cutoff) model $^{2}$ [11]. The Fermi-LAT pion-decay template is taken from the reference Galactic model in [9]. We extract just the pion-decay component of this model and use it as a template for neutrino emission. We do not convert the gamma-ray flux into an absolute prediction of the number of neutrinos but only consider the spatial distribution of the emission. The Fermi-LAT energy range where the model is validated is substantially lower than the energies of the neutrinos to which we are sensitive. A hardening in the spectrum of the cosmic rays could substantially increase the neutrino predictions.

In [11] the authors noticed that the model above does, in fact, under-predict the flux of gamma rays above a few $\mathrm{GeV}$ in the Galaxy, especially for higher-energy observations of the H.E.S.S and Milagro collaborations. The same authors were able to explain this residual flux by allowing the diffusion coefficient to vary depending on the Galactic radius. The result of this study is the KRA- $\gamma$ model, which matches the gamma-ray data quite closely. Since the KRA- $\gamma$ model is based on the same underlying model of the interstellar medium (ISM) as the Fermi-LAT pion-decay template, the spatial features are very similar between the two models, with KRA- $\gamma$ being brighter at the Galactic center.

\section{Analysis Methods}

\subsection{Unbinned Maximum Likelihood Spatial Template Analysis}

The unbinned method used in this work is a modification of the unbinned maximum likelihood analysis commonly employed in IceCube point-source searches [12,6]. The first modification is to account for the extension of the source by mapping the changing detector acceptance and convolving the extended source hypothesis (in contrast with the delta-function source hypothesis used in point-source searches) with the PSF of the events. The other modification relates to the estimate of the background using data. In a point-source analysis, a hypothetical source has a very small contribution to it's declination band and is treated as negligible for determining the background. For the Galactic plane, the signal may extend over the entire sky and is no longer negligible and so a different likelihood formalism is needed. The signal-subtracted likelihood acknowledges this contribution by making a small correction to the method as introduced in [13]:

\footnotetext{
${ }^{2}$ The conventional KRA diffusion model is defined in [10]
} 


$$
L\left(n_{s}, \gamma\right)=\prod_{i=1}^{N}\left(\frac{n_{s}}{N} S_{i}\left(\mathbf{x}_{i}, \sigma_{i}, E_{i} ; \gamma\right)+\tilde{D}_{i}\left(\delta_{i}, E_{i}\right)-\frac{n_{s}}{N} \tilde{S}_{i}\left(\delta_{i}, E_{i}\right)\right)
$$

where $n_{s}$ is the number of signal events for a flux following spectral index $\gamma ; N$ is the total number of events in the sample; $S_{i}\left(\mathbf{x}_{i}, \sigma_{i}, E_{i} ; \gamma\right)$ is the signal probability distribution function (PDF) for event $i$ at equatorial coordinates $\mathbf{x}_{i}=\left(\alpha_{i}, \delta_{i}\right)$ with Gaussian PSF of width $\sigma_{i}$ and energy estimator $E_{i} ; \tilde{D}_{i}$ is defined as:

$$
\tilde{D}_{i}\left(\delta_{i}, E_{i}\right)=\frac{n_{s}}{N} \tilde{S}_{i}\left(\delta_{i}, E_{i}\right)+\left(1-\frac{n_{s}}{N}\right) B_{i}\left(\delta_{i}, E_{i}\right),
$$

where $\tilde{S}_{i}$ is constructed by integrating $S_{i}$ in a small declination bin $\left(\sim 1^{\circ}\right)$ over right ascension; $B_{i}$ is the background PDF.

The $S_{i}$ terms, which encode information about both the raw signal expectation and the detector performance, are obtained by convolving the neutrino flux model with the effective area obtained from detailed simulations (cf. [4]). The resulting map is finally convolved with the PSF for each event, modeled by a Gaussian distribution of width $\sigma_{i}$ estimated for each event and ranging from $0.1^{\circ}$ to $3.0^{\circ}$.

A nested log-likelihood ratio between the best-fit signal strength and the null hypothesis (no Galactic component) is used to construct a test statistic. Under the null hypothesis and in the large sample limit, the test statistic follows a half- $\chi^{2}$-distribution as expected from [14]. Final upper limits, sensitivities (given as median upper limits) and significances, such as the p-value of the observed test statistic, are always calculated using background trials obtained from scrambled data. We use a $90 \%$ Neyman upper limit construction.

The data sample for this analysis is described in [6]. It is an all-sky sample that spans seven years with a total live time of 2431 days and 730130 events. The sensitivity comes primarily from the northern sky, where IceCube sees a wide energy range of muons induced by neutrinos. Though the sample extends into the southern sky, the sensitivity there is limited to very high energies since energy selection and veto techniques are used that reject the softer background of down-going muons from cosmic-ray air showers.

\subsection{Binned Maximum Likelihood Spatial Template Analysis}

The binned maximum likelihood analysis is an extension of the analysis presented in [4]. In this analysis, the contributions from conventional atmospheric neutrinos [15], prompt atmospheric neutrinos [16] and isotropic astrophysical neutrinos with a power-law energy spectrum are fitted to experimental data. The events are binned according to the reconstructed zenith angle and an energy estimator. The resulting histograms are analyzed using a maximum-likelihood approach. Each bin is modeled by a Poisson likelihood function:

$$
L_{i}(\theta, \xi)=e^{-\mu_{i}(\theta, \xi)} \cdot \frac{\mu_{i}^{k}}{k !}
$$

where $\theta=\theta\left(\Phi_{\text {astro }}, \gamma_{\text {astro }}, \ldots\right)$ describes the signal parameters (i.e. properties of the astrophysical fluxes) and $\xi$ describe the nuisance parameters. The expected number of events in bin $i, \mu_{i}$, is given by the sum of the four flux expectations for the conventional, prompt, isotropic astrophysical and 
Galactic flux:

$$
\begin{aligned}
\mu_{i}(\theta, \xi) & =\mu_{i}^{\text {conv }}\left(\xi_{\text {conv }}, \xi_{\text {det }}\right)+\mu_{i}^{\text {astro }}\left(\Phi_{\text {astro }}, \gamma_{\text {astro }}, \xi_{\text {det }}\right) \\
& +\mu_{i}^{\text {prompt }}\left(\xi_{\text {prompt }}, \xi_{\text {det }}\right)+\mu_{i}^{\text {Galactic }}\left(\Phi_{\text {Galactic }}, \xi_{\text {det }}\right),
\end{aligned}
$$

where $\xi_{\text {conv }}$ and $\xi_{\text {prompt }}$ refer to nuisance parameters taking into account the theoretical uncertainties on the respective fluxes and $\xi_{\operatorname{det}}$ refers to nuisance parameters taking into account detector uncertainties. For more information on these parameters we refer to [4]. The final, global likelihood is the product of all per-bin likelihoods $L=\prod_{i} L_{i}$.

Compared to [4], this analysis is extended by including the reconstructed right ascension, thus changing the histograms from two to three dimensions. Additionally, a template for the Galactic contribution, $\mu^{\text {Galactic }}$, is added to the fit. Note that for the Fermi-LAT $\pi^{0}$-decay template the Galactic spectral index is an additional signal parameter free to float in the fit. In contrast to the method described in the previous section, this method models the expected contributions of every flux component using Monte Carlo simulations. This allows us to see how the isotropic component changes with the best-fit Galactic component. The test statistic is defined as a log-likelihood ratio in the same fashion as the previous method, with the same limit and significance calculations.

The data sample for this is described in [4]. Compared to the previous method, the sample has a significantly higher muon neutrino purity of $>99.7 \%$ with comparable effective area and a slightly improved PSF. The sample is, however, limited to the northern hemisphere where the high neutrino purity standards can be achieved. The time period is somewhat smaller as this selection does not apply to the time period when IceCube had just 40 of the final 86 strings deployed. The data set spans six calendar years with a total live time of 2060 days and 354792 events.

\section{Results}

\subsection{Constraints on Diffuse Emission in the Plane}

The sensitivities and results of spatial template analyses are summarized in Table 1. Some excess from the Galaxy is observed in all cases, though it is not statistically significant. Because of the somewhat better sensitivity, the unbinned method was assigned, in advance of unblinding the data, to be the main result, with the binned method acting as a cross check. The upper limit for the KRA- $\gamma$ test is shown in Figure 2 in comparison to the ANTARES upper limit, the KRA family of predictions, and the isotropic diffuse neutrino flux.

Table 1: Summary of results for both Galactic plane analysis methods. The sensitivities and upper limits for KRA- $\gamma$ are given in units of the model prediction. For the Fermi $\pi^{0}$-decay template they are given in units of a $\left(E_{n u} / 100 T e V\right)^{-2.5} v_{\mu}+\bar{v}_{\mu}$ flux.

\begin{tabular}{c|c|c|c||c|c|c} 
& \multicolumn{3}{|c||}{ unbinned method } & \multicolumn{3}{c}{ binned method (cross check) } \\
Spatial Template & p-value & Sens. $\phi_{90 \%}$ & U.L. $\phi_{90 \%}$ & p-value & Sens. $\phi_{90 \%}$ & U.L. $\phi_{90 \%}$ \\
\hline$\pi^{0}$-decay, $E^{-2.5}$ & $37 \%$ & $2.97 \times 10^{-18}$ & $3.83 \times 10^{-18}$ & $7.0 \%$ & $3.16 \times 10^{-18}$ & $6.13 \times 10^{-18}$ \\
KRA- $\gamma(50 \mathrm{PeV})$ & $29 \%$ & $79 \%$ & $120 \%$ & $6.9 \%$ & $95 \%$ & $170 \%$ \\
\hline
\end{tabular}




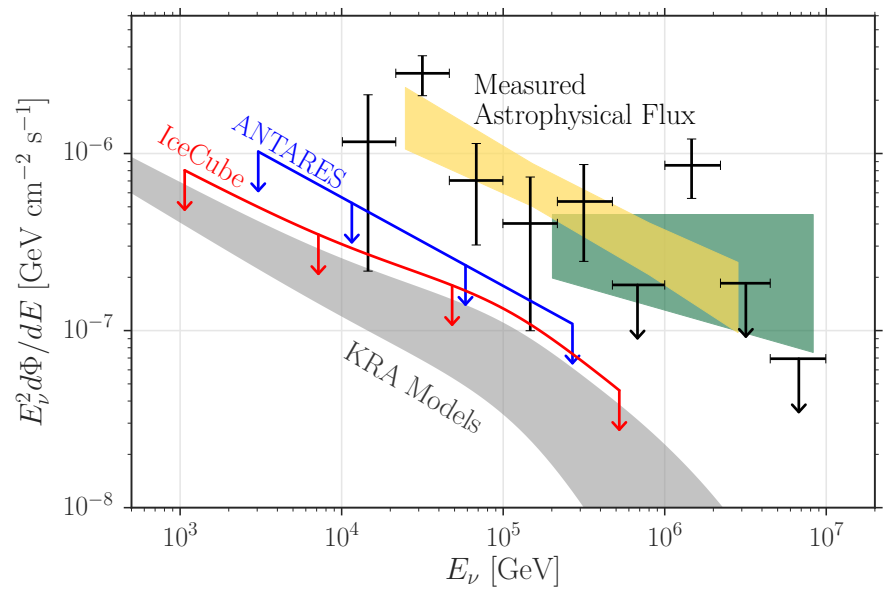

Figure 2: Upper limits on the three-flavor (1:1:1 flavor ratio assumption) neutrino flux from the Galaxy with respect to KRA model predictions and the measured astrophysical neutrino flux. The limit for the KRA- $\gamma(50 \mathrm{PeV})$ model test is shown in red. The energy range of validity is from $1 \mathrm{TeV}$ to $500 \mathrm{TeV}$. This range is calculated by finding the high and low energy thresholds where removing simulated signal events outside these values decreases the sensitivity by 5\% each. The ANTARES limit is shown in blue [8]. The range of predictions for all KRA models [11] is shown as the gray band. For comparison, measurements of the all-sky diffuse flux are shown: a differential unfolding (black points) and a power-law unfolding (yellow band) of combined IceCube data sets from [5] as well as a measurement based only on northern sky muon data (green band) from [4].

The Galactic excesses are somewhat larger and more significant for the cross check, and this difference was investigated carefully for the benchmark Fermi pion decay template. Part of the difference comes from the additional year of data used by the unbinned method. If we restrict this method to the same time period, the p-value drops from $37 \%$ to $22 \%$. Running the unbinned method on the alternative sample yields a p-value of $21 \%$. Due to the purity requirement for modeling the background with simulation, the check of the binned method on the alternative data set is not possible.

\subsection{D Likelihood Scan and Implications for the Isotropic Astrophysical Flux}

Using the binned method a two-dimensional profile likelihood scan of the Galactic normalization and Galactic spectral index is performed for the benchmark Fermi-LAT pion-decay template. The results of this scan are shown in Figure 3 (left). The best-fit spectral index is $\gamma_{\text {Galactic }}=2.07$, and the best-fit normalization at $100 \mathrm{TeV}$ is $\Phi_{\text {Galactic }}=3.13 \cdot 10^{-18} \mathrm{GeV}^{-1} \mathrm{~cm}^{-2} \mathrm{~s}^{-1}$ (the same units and reference energy are used in Table 1). The confidence contours have been estimated using Wilks' Theorem [17], which has been cross-checked at several points in the parameter space with Monte Carlo pseudo-experiments. The best-fit spectral index is harder than expected from a naive extrapolation of the pion template. However, it is consistent with an index of 2.5 at the $1.65 \sigma$ level.

Since the binned method is an extension of the method used to characterize the isotropic astrophysical flux in [4], we can analyze the impact of allowing an additional Galactic component in the fit on the isotropic flux parameters. For this we performed a profile-likelihood scan of the isotropic flux normalization and spectral index while allowing the Galactic flux parameters to float 
freely at every scan point. Figure 3 (right) shows the resulting likelihood contours in comparison to the contours obtained by restricting the Galactic flux to zero. The color scale shows the best-fit Galactic plane spectral index at each point in the scan. Although the additional freedom given by the Galactic flux nuisance causes the isotropic normalization to decrease, the size of the contour grows only marginally. The hypothesis of zero isotropic flux is still excluded with $>3 \sigma$ significance. This shows that the observation of an isotropic astrophysical signal is robust against a signal from the Galactic plane and that the latter can only contribute a sub-dominant fraction to the total observed astrophysical flux.
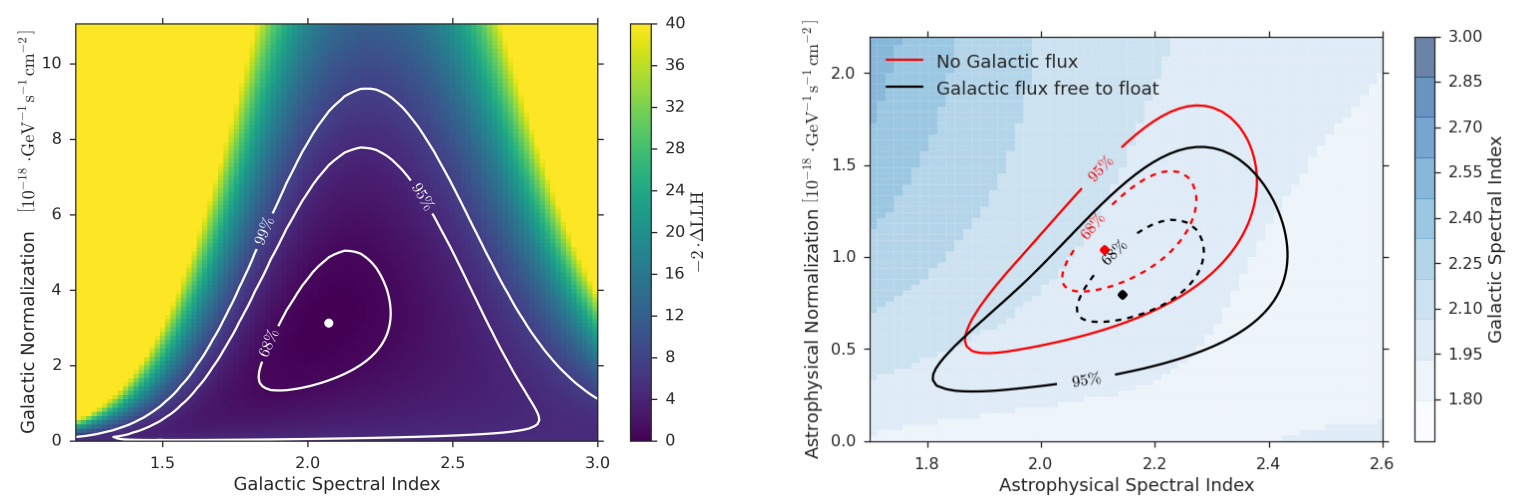

Figure 3: Left: 2D likelihood scan of Galactic flux normalization and Galactic spectral index. Right: Contours of isotropic astrophysical flux normalization and spectral index with and without Galactic flux.

\section{Conclusions}

We have presented searches for neutrino signals associated with the Galactic plane using seven years of IceCube muon neutrino data, focusing on diffuse emission from interactions of cosmic rays with the ISM. We are able to exclude that more than $14 \%$ of the diffuse neutrino flux as measured in [5] comes from the Galactic plane for the case of the Fermi-LAT template and an $E^{-2.5}$ power law.

Our measurement is primarily sensitive in the northern hemisphere, where IceCube has a high efficiency for a wide energy range of muons induced by neutrinos. Our limits are quoted assuming various all-sky spatial models of neutrino emission. The most optimistic, KRA- $\gamma$ with a $50 \mathrm{PeV}$ cosmic-ray cutoff, concentrates the most flux towards the Galactic center. Even though a higher fraction of the flux is in the southern sky, our limits are just $20 \%$ higher than this model prediction.

The ANTARES detector also sets relevant constraints measured directly in the region surrounding the Galactic center $\left(-40^{\circ}<l<40^{\circ}\right.$ and $\left.-3^{\circ}<b<3^{\circ}\right)$. They limit the neutrino flux to be less than $60 \%$ higher than the KRA- $\gamma$ model at $100 \mathrm{TeV}$ [8].

While our flux constraints focus on the plane of the Galaxy, there are still possibilities for the flux to originate in or very near the Galaxy. The possibility of cosmic-ray interactions with a gas halo extending out to $\sim 100 \mathrm{kpc}$ is still actively explored [18, 19, 20]. Another possibility would be the annihilation [13] or decay [21] of dark matter particles in the Galactic halo. For these hypotheses, the emission is much more isotropic than the Galactic emission templates that we tested. 
There are possibilities to improve the sensitivity for Galactic neutrino searches. A search for point sources in the southern hemisphere using cascade-like events in IceCube has been shown to have a sensitivity comparable to ANTARES [22]. A joint analysis that includes both the track and cascade channels will offer promising improvements. Additionally, a joint Galactic plane analysis between IceCube and ANTARES, similar to the joint point-source analysis that produced excellent limits in the southern sky [23], would provide the strongest constraints on neutrinos from the Milky Way with all available data.

\section{References}

[1] G. W. Clark, G. P. Garmire, and W. L. Kraushaar, ApJL 153 (1968) L203.

[2] IceCube Collaboration, M. G. Aartsen et al., Science 342 (2013) 1242856.

[3] IceCube Collaboration, M. G. Aartsen et al., Phys. Rev. Lett. 113 (2014) 101101.

[4] IceCube Collaboration, M. G. Aartsen et al., Astrophys. J. 833 (2016) 3.

[5] IceCube Collaboration, M. G. Aartsen et al., Astrophys. J. 809 (2015) 98.

[6] IceCube Collaboration, M. G. Aartsen et al., Astrophys. J. 835 (2017) 151.

[7] A. Neronov and D. Semikoz, Phys. Rev. D93 (2016) 123002.

[8] S. Adrián-Martínez et al., Physics Letters B 760 (2016) 143-148.

[9] M. Ackerman et al., ApJ 750 (2012) 3.

[10] C. Evoli et al., Phys. Rev. D85 (2012) 123511.

[11] D. Gaggero et al., ApJL 815 (2015) L25.

[12] J. Braun et al., Astroparticle Physics 29 (2008) 299-305.

[13] IceCube Collaboration, M. G. Aartsen et al., Eur. Phys. J. C75 (2015) 492.

[14] G. Cowan et al., Eur. Phys. J. C71 (2011) 1554. [Erratum: Eur. Phys. J.C73,2501(2013)].

[15] M. Honda et al., Phys. Rev. D 75 (2007) 043006.

[16] R. Enberg, M. H. Reno, and I. Sarcevic, Phys. Rev. D 78 (2008) 043005.

[17] S. S. Wilks, Ann. Math. Statist. 9 (1938) 60-62.

[18] R. Feldmann, D. Hooper, and N. Y. Gnedin, ApJ 763 (2013) 21.

[19] A. M. Taylor, S. Gabici, and F. Aharonian, $P h R v D 89$ (2014) 103003.

[20] O. Kalashev and S. Troitsky, PhRvD 94 (2016) 063013.

[21] K. Murase, R. Laha, S. Ando, and M. Ahlers, PhRvL 115 (2015) 071301.

[22] IceCube Collaboration, M. G. Aartsen et al., arXiv:1705.02383.

[23] S. Adrian-Martinez et al., Astrophys. J. 823 (2016) 65. 